\title{
A Distributional Approach to Conditionally Convergent Series
}

\author{
Gregory A. Ciccarelli \\ Schreyer Honors College \\ The Pennsylvania State University \\ 10 Schreyer Honors College \\ University Park, Pennsylvania 16802-3905 USA \\ Patrick Moylan \\ Physics Department \\ Abington College \\ The Pennsylvania State University \\ Abington, Pennsylvania 19001 USA
}

Received: April 4, $2008 \quad$ Accepted: September 1, 2008

\begin{abstract}
Whether the car's gas tank is filled up on Monday and the paycheck is deposited on Tuesday, or vice versa, the contribution of those two transactions to the checkbook's final balance is the same. By the commutative property, order does not matter for the algebraic addition of a finite number of terms. However, for a super banker who conducts an infinite number of transactions, order may matter. If a series (sum of all transactions/terms) is convergent and the order of term does not matter, then the series is absolutely convergent. If a series is convergent but the order of terms does matter, then it is conditionally convergent. Georg Bernhard Riemann proved the disturbing result that the final sum of a conditionally convergent series could be any number at all or divergent. In two, three and higher dimensions, the matter is even worse, and such series with double and triple sums are not even well-defined without first giving sum interpretation to the (standard) order in which the series is to be summed, e.g., in three dimensions, summing over expanding spheres or expanding cubes, whose points represent ordered triples occurring in the summation. In this note we show using elementary notions from distribution theory that an interpretation exists for conditionally convergent series so they have a precise, invariant meaning.
\end{abstract}

\section{INTRODUCTION}

In this note we show using elementary notions from distribution theory that an interpretation exists for conditionally convergent series so that they have a precise, invariant meaning. We think our method to be sufficiently elementary that it can be understood by undergraduate mathematics and science majors, and we advocate mentioning this approach when discussing conditionally convergent series in the calculus curriculum. Our results also demonstrate the power and relevance of distribution theory in such problems.
Basically our idea is as follows: we view a conditionally convergent series in a distributional sense on a space of square integrable sequences. Integration of the distribution against a suitable test function results in an absolutely convergent series. The example used in this note will be the alternating harmonic series, which is essentially the Madelung constant of a onedimensional chain of ions with alternating charges [1].

We start with a test function in terms of the variable of summation $(n)$ and which depends upon a parameter $\alpha$. A test function must go to zero faster than any 
polynomial goes to zero in the limit as $n$ approaches infinity. In addition, we require that the limit of the test function must be 1 as $\alpha$ approaches zero. $e^{-\alpha n}(\alpha>0)$ is such a test function. We now define the distribution to be the original conditionally convergent sequence function, then multiply the test function against this distribution and sum over $n$, i.e. we complete the scalar product of the distribution with the test function. The resulting series with the modified sequence function is now absolutely convergent. For the alternating harmonic series example, the new series is absolutely convergent for $\alpha>0$ by the ratio test for absolutely convergence. Finally, $\alpha$ is allowed to approach zero. Allowing $\alpha$ to approach zero seems to turn the modified series back into the original alternating harmonic series, but this can only be true if the conditions of the Lebesgue dominated convergence theorem or the monotone convergence theorem are satisfied. In general, multiplying the test function against the original sequence function and then summing fundamentally changes the original series. The resulting function returns the same sum as the original series for the natural term order of the original series, yet the terms in the new series can be rearranged without affecting the final sum. In other words, we keep the desired aspects of conditional convergence without having to deal with the perplexing and troublesome aspects, such a Riemann's result. By defining conditionally convergent series in the manner advocated in this article, they can be used without ambiguity and without additional restrictions in fields that involve them, like solid state physics or chemistry or quantum physics. We believe the approach advocated in this article makes for a much clearer and precise treatment of topics like Madelung sums in physics and chemistry [1], and, hence, students should be made aware of this approach as early as possible in their educations.

\section{SOME STANDARD RESULTS ON CONDITIONAL AND ABSOLUTE CONVERGENCE OF SERIES}

Definition 1 (Ref. [2], p. 59). Let $\left\{a_{n}\right\}$ be a sequence, and let $S_{n}=a_{1}+a_{2}+\ldots+a_{n}$ be the $n^{\text {th }}$ partial sum. If $S_{n}$ exists in the limit of $\mathrm{n} \rightarrow \infty$, then we say that

$$
\sum_{n=1}^{\infty} a_{n}
$$

Is a convergent series, and write

$$
\lim _{n \rightarrow \infty} S_{n}=\sum_{n=1}^{\infty} a_{n} .
$$

Thus a series is convergent if and only if its sequence of partial sums is convergent. The limit of the sequence of partial sums is the sum of the series. A series which is not convergent is a divergent series.

Definiton 2 (Ref. [2], p. 71). The series $\sum_{n=1}^{\infty} a_{n}$ is absolutely convergent if and only if the series $\sum_{n=1}^{\infty}\left|a_{n}\right|$ is convergent.

Definition 3 (Ref. [3]). The series $\sum_{n=1}^{\infty} a_{n}$ is conditionally convergent if and only if the series $\sum_{n=1}^{\infty} a_{n}$ is convergent but not absolutely convergent.

Proposition (Ref. [2], p. 71). An absolutely convergent series is convergent.

Proof. Assume that $\sum_{n=1}^{\infty} a_{n}$ is absolutely convergent and define

$$
\begin{aligned}
& a_{n}^{+}=\left\{\begin{array}{c}
a_{n} \text { if } a_{n}>0 \\
0 \text { if } a_{n}<0
\end{array}\right. \\
& a_{n}^{-}=\left\{\begin{array}{c}
\left|a_{n}\right| \text { if } a_{n}<0 \\
0 \text { if } a_{n}>0
\end{array}\right.
\end{aligned}
$$

Clearly, since $\sum_{n=1}^{\infty}\left|a_{n}\right|$ is convergent by definition, we have that $\sum_{n=1}^{\infty} a_{n}^{+}$and $\sum_{n=1}^{\infty} a_{n}^{-}$ are convergent series of positive terms. Thus

$$
\sum_{n=1}^{\infty} a_{n}=\sum_{n=1}^{\infty} a_{n}^{+}-\sum_{n=1}^{\infty} a_{n}^{-}
$$


is also convergent.

Theorem (Ref. [2], p. 71, Leibniz). Let $\left\{a_{n}\right\}$ be a decreasing sequence of positive terms such that $a_{n} \rightarrow 0$ as $n \rightarrow \infty$. Then the series

$$
\sum_{n=1}^{\infty}(-1)^{n+1} a_{n}
$$

is convergent.

Proof. Write $S_{n}$ for the $n^{\text {th }}$ partial sum of the series $(-1)^{n+1} a_{n}$. By definition we have

$$
\begin{gathered}
S_{2 n+1}=a_{1}-a_{2}+a_{3}-\ldots+a_{2 n-1}-a_{2 n}+a_{2 n+1} \\
S_{2 n-1}=a_{1}-a_{2}+a_{3}-\ldots+a_{2 n-1}
\end{gathered}
$$

and so subtracting we have

$$
S_{2 n+1}=S_{2 n-1}-a_{2 n}+a_{2 n+1}
$$

Since $\left\{a_{n}\right\}$ is a decreasing sequence, $a_{2 n}>$ $a_{2 n+1}$ and so $S_{2 n+1}<S_{2 n-1}$. Thus we have a decreasing sequence.

$$
S_{1}>S_{3}>S_{5}>\ldots>S_{2 n-1}>S_{2 n+1}>\ldots
$$

Similarly, $S_{2 n}>S_{2 n-2}$ and we have an increasing sequence

$$
S_{2}<S_{4}<S_{6}<\ldots<S_{2 n-2}<S_{2 n}<\ldots
$$

Also $S_{2 n+1}=S_{2 n}+a_{2 n+1}>S_{2 n}$. Thus $S_{2}<S_{4}$ $<S_{6}<\ldots<S_{2 n-2}<S_{2 n}<S_{2 n+1}<S_{2 n-1}<\ldots<S_{5}$ $<S_{3}<S_{1}$ and the sequence $S_{1}, S_{3}, S_{5} \ldots$ is a decreasing sequence which is bounded below (by $S_{2}$ ), and is convergent to a (say). Similarly $S_{2}, S_{4}, S_{6} \ldots$ is an increasing sequence which is bounded above (by $S_{1}$ ) and is convergent to $\beta$ (say). Also

$$
S_{2 n+1}-S_{2 n}=a_{2 n+1}
$$

and so letting $n \rightarrow 0$ we have

$$
\alpha-\beta=0 \text {. }
$$

So $\alpha=\beta$ and all the partial sums are tending to $\alpha$, so the series converges.

Corollary (Ref. [2], p. 72). The alternating harmonic series is conditionally convergent. Proof. We have

$$
\sum_{n=1}^{\infty}\left|\frac{(-1)^{n+1}}{n}\right|=\sum_{n=1}^{\infty} \frac{1}{n}
$$

and this is the harmonic series, which is clearly divergent [4]. Thus the series is not absolutely convergent. We now show using the above Theorem that this series is still convergent, and so is conditionally convergent. Write $a_{n}=(1 / n)$, so $a_{n}>0, a_{n+1}$ $<a_{n}$, and $a_{n} \rightarrow 0$ as $n \rightarrow \infty$. Thus all the conditions of Leibniz's theorem are satisfied, and so the series is convergent.

Proposition 2 (Ref. [2], p. 78): Rearranging absolutely converging series.

Let $\sum a_{n}$ be an absolutely convergent series and suppose that $\left\{b_{n}\right\}$ is a rearrangement of $\left\{a_{n}\right\}$. Then $\sum b_{n}$ is convergent, and $\sum b_{n}=\sum a_{n}$.

Proof. First suppose that $\sum a_{n}$ consists only of positive terms. Consider the partial sum,

$$
S_{n}=\sum_{v=1}^{n} a_{v} .
$$

All terms of $S_{n}$ occur in the $m^{\text {th }}$ partial sum of the rearranged series

$$
S_{m^{\prime}}=\sum_{v=1}^{m} b_{v}
$$

provided $\mathrm{m}$ is large enough. Hence, $S_{m}$ > $S_{n}$. On the other hand, we may determine an index $n$ ' such that the partial sum

$$
S_{n^{\prime}}=\sum_{v=1}^{n^{\prime}} a_{v}
$$

of the first series contains all terms $b_{1}, b_{2}$, $b_{3} \ldots b_{m}$. It thus follows that $S_{n^{\prime}} \geq S_{m^{\prime}} \geq S_{n}$. Since $S_{n}$, and $S_{n}$ converge to the same value, $S_{n}$, and $S_{n}$ can be made to differ from each other by an arbitrarily small amount. This fact together with the just established inequality implies that the rearranged series also converges to the same limit as the original series. If the absolutely convergent series has both positive and negative terms, we may regard it as the difference of two series each of which has only positive terms. Since, in the rearrangement of the original series, each of these two series merely undergoes rearrangement. They therefore converge to the same value as before, and the same is true of the original series when rearranged.

Proposition 3 (Ref. [2], p. 76, Riemann). Let $a_{n}$ be a conditionally convergent series. 
Choose $x \in \mathrm{R}$. Then there is a rearrangement $\left\{b_{n}\right\}$ of $\left\{a_{n}\right\}$ such that $b_{n}=x$. In other words, we can rearrange a conditionally convergent series to get any answer we want! It is this lack of invariance under rearrangement of terms which makes conditionally convergent series imprecise and confusing, and which requires specifying further conditions on the series, such as the order in which they are to be summed.

Rather than prove the theorem we shall illustrate it with a detailed example which shows the essence of the proof. We consider the alternating harmonic series and show that different groupings of terms give different partial sums that converge to different values. The alternating harmonic series in standard order is

$$
\sum_{k=1}^{\infty} \frac{(-1)^{k+1}}{k}=1-\frac{1}{2}+\frac{1}{3}-\frac{1}{4}+\ldots
$$

and is equal to $\ln (2)$, as we will show below. We will say that a series is a simple $(p, n)$ rearrangement of the alternating harmonic series if the first term is 1 , the subsequence of positive terms and the subsequence of negative terms are in original order, and the series consists of blocks of $p$ positive terms followed by $n$ negative terms, i.e., the series has the form

$$
\begin{aligned}
& \left(1+\frac{1}{3}+\frac{1}{5}+\ldots+\frac{1}{(2 p-1)}\right)-\left(\frac{1}{2}+\frac{1}{4}+\ldots+\frac{1}{(2 n)}\right)+ \\
& +\left(\frac{1}{(2 p+1)}+\ldots+\frac{1}{(4 p-1)}\right)-\left(\frac{1}{(2 n+2)}+\ldots+\frac{1}{(4 n)}\right)+\ldots
\end{aligned}
$$

For example, with $p=1$ and $n=2$ the series becomes

$$
\begin{gathered}
1-\frac{1}{2}-\frac{1}{4}+\frac{1}{3}-\frac{1}{6}-\frac{1}{8}+\frac{1}{5}-\frac{1}{10}-\frac{1}{12} \\
\quad+\frac{1}{7}-\frac{1}{14}-\frac{1}{16}+\ldots \\
=\left(1-\frac{1}{2}\right)-\frac{1}{4}+\left(\frac{1}{3}-\frac{1}{6}\right)-\frac{1}{8}\left(\frac{1}{5}-\frac{1}{10}\right) \\
\quad-\frac{1}{12}\left(\frac{1}{7}-\frac{1}{14}\right)-\frac{1}{16}+\ldots \\
=\frac{1}{2}-\frac{1}{4}+\frac{1}{6}-\frac{1}{8}+\frac{1}{10}-\frac{1}{12}+\frac{1}{14}-\frac{1}{16}+\ldots \\
=\frac{1}{2}\left[1-\frac{1}{2}+\frac{1}{3}-\frac{1}{4}+\frac{1}{5}-\frac{1}{6}+\frac{1}{7}-\frac{1}{8}+\ldots\right] \\
=\frac{1}{2} \ln (2)
\end{gathered}
$$

using the shortly to be proved fact that the sum of the alternating harmonic series in standard order in In(2) [c.f., Corollary below]. From this calculation, one readily sees that a different sum may be obtained by rearranging the original series.

We now prove that a rearrangement of the form in eqn. (10) has the sum [5]

$$
\ln (2)+\frac{1}{2} \ln \left(\frac{p}{n}\right) \text {. }
$$

Suppose $\quad \sum_{k=1}^{\infty} a_{k}$ is a simple $(\mathrm{p}, \mathrm{n})$ rearrangement of the form given in eqn. (10). For each positive integer $m$ define

and

$$
H_{m}=1+\frac{1}{2}+\frac{1}{3}+\ldots+\frac{1}{m}
$$

$$
E_{m}=H_{m}-\ln (m+1) \text {. }
$$

The term $E_{m}$ is the error of the Riemann sum $H_{m}$ illustrated in Figure 1 for approximating the definite integral

$$
\int_{1}^{m+1} \frac{1}{x} d x=\ln (m+1) .
$$

The sequence of error terms is a bounded increasing sequence of positive numbers whose limit is $\gamma$, the Euler constant [6]. ${ }^{1}$ By unraveling the rearrangement we get that

$$
\begin{gathered}
\sum_{k=1}^{m(p+n)} a_{k}=\sum_{j=1}^{m}\left[\sum_{i=p(j-1)+1}^{p j} \frac{1}{2 i-1}-\sum_{i=n(j-1)+1}^{n j} \frac{1}{2 i}\right] \\
=\sum_{i=1}^{2 m p-1} \frac{1}{i}-\frac{1}{2} \sum_{i=1}^{m p-1} \frac{1}{i}-\frac{1}{2} \sum_{i=1}^{m n} \frac{1}{i} \\
=H_{2 m p-1}-\frac{1}{2} H_{m p-1}-\frac{1}{2} H_{m n} \\
=\ln (2 m p)-\frac{1}{2} \ln (m p)-\frac{1}{2} \ln (m n+1) \\
+E_{2 m p-1}-\frac{1}{2} E_{m p-1}-\frac{1}{2} E_{m n} \\
=\ln (2)+\frac{1}{2} \ln \left(\frac{m p}{m n+1}\right) \\
+E_{2 m p-1}-\frac{1}{2} E_{m p-1}-\frac{1}{2} E_{m n}
\end{gathered}
$$

1 The Euler-Mascheroni constant, as it is sometimes called, is the difference between the harmonic series and the natural logarithm,

$$
\gamma=\lim _{n \rightarrow \infty}\left[\sum_{k=1}^{n} \frac{1}{k}-\ln (n)\right],
$$

which, if we recall that $\int_{1}^{\infty} \frac{d x}{x}=\lim _{n \rightarrow \infty} \ln (n)$, is exactly the limit of $E_{m}$ as $m \rightarrow \infty$. One of the, as of yet, unsolved problems in mathematics is whether or not $\gamma$ is rational [7]. 


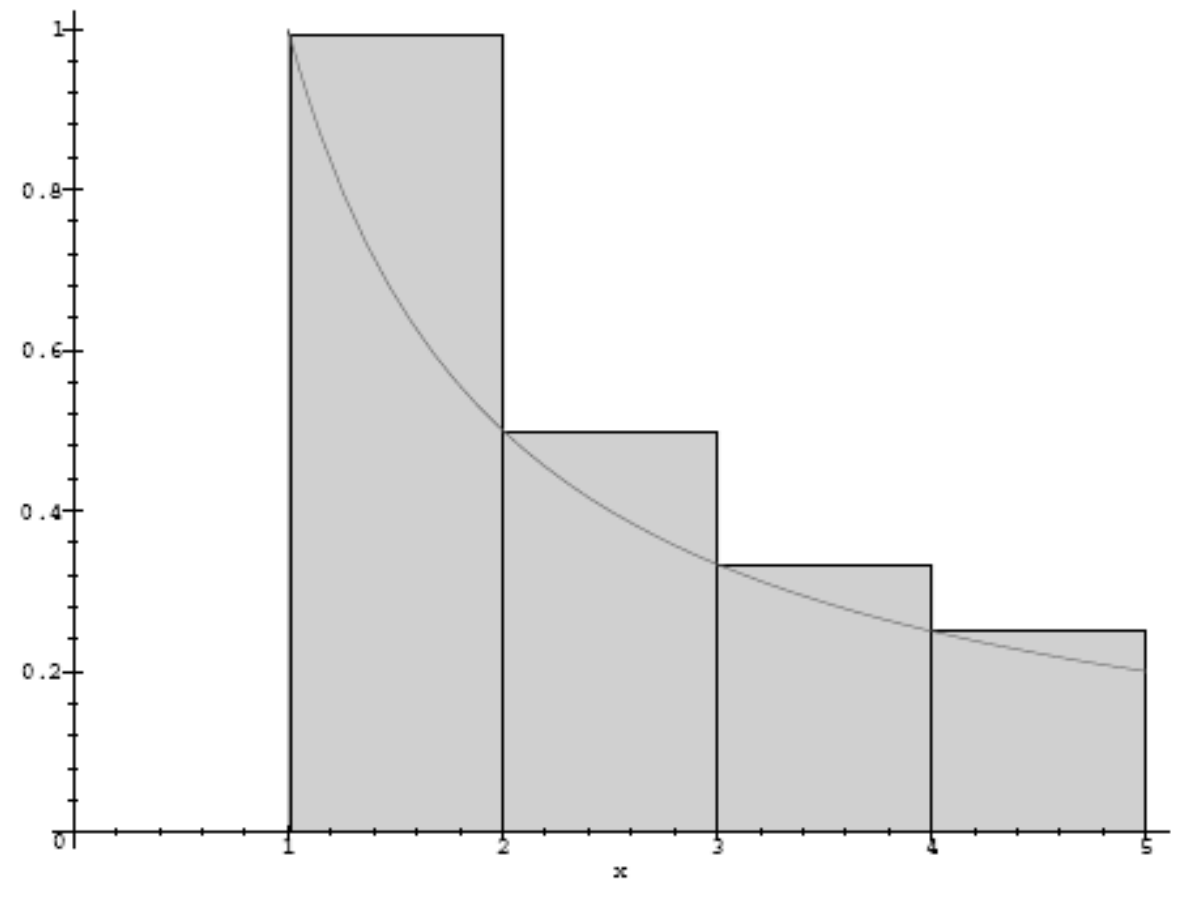

Figure 1. $H_{m}$ and $E_{m}$ for $m=4 . H_{m}$ is the sum of areas of the rectangles, and $E_{m}$ is the sum of the areas of the triangular regions above the graph of $1 / x$. That is, $E_{m}$ is the error of the upper Riemann sum $H_{m}$.

Now let $m$ go to infinity to obtain

$$
\begin{gathered}
\lim _{m \rightarrow \infty} \sum_{k=1}^{m(p+n)} a_{k}=\ln (2)+\frac{1}{2} \ln \left(\frac{p}{n}\right)+\gamma-\frac{1}{2} \gamma-\frac{1}{2} \gamma \\
=\ln (2)+\frac{1}{2} \ln \left(\frac{p}{n}\right) .
\end{gathered}
$$

This considers only those partial sums with a full complement of $p+n$ terms, which is sufficient to give the sum of the series, since the terms approach zero. Finally, we use the fact that the rational numbers are dense in the reals to see that it is possible to obtain a rearrangement of the alternating harmonic series that can be made arbitrarily close to any real number we choose.

Note that when $p=n=1$ we get $\ln (2)$ for the natural term order, i.e, the alternating harmonic series in standard order equals $\ln (2)$.

Corollary (Ref. [3]). The sum of the alternating harmonic series in natural term order is $\ln (2)$.

\section{A DISTRIBUTIONAL APPROACH}

We now introduce the Schwartz space, $S$, on R: $S$ consists of all infinitely differentiable functions $\varphi(x)$ and all of its derivatives go to zero as $x$ goes to infinity faster than any polynomial, i.e.,

$$
p(x) \frac{d^{n}}{d x^{n}} \varphi(x) \rightarrow 0 \text { as } x \rightarrow \infty .
$$

Let $S$ be the space of all continuous linear functionals of $S$. For $f$ in $S$ and $\varphi$ in $S$, we write $\langle f, \varphi\rangle$ for the linear functional on $S$. $S$ is the space of tempered distributions. Any function $f(x)$ on $\mathrm{R}$ gives rise to a distribution on $S$ by the formula

$$
\langle f, \varphi\rangle=\int_{\mathrm{R}} f(x) \varphi(x) d x .
$$


Now to our example with the alternating harmonic series. Let $\alpha$ be a positive real number, and define:

$$
\varphi_{\alpha}(x)=\left\{\begin{array}{ll}
e^{-\alpha x} & x \geq 0 \\
0 & x<0
\end{array} .\right.
$$

It is easy to see that $\varphi$ is in the Schwartz space [8]. We define the distribution

$$
f(x)=\sum_{n=1}^{\infty} \frac{(-1)^{n+1}}{n} \delta(x-n),
$$

where $\delta(x-n)$ is the Dirac delta function [8]. (The Dirac delta function acts on $\varphi \in S$ via eqn. (15) (c.f. Ref. [8]). This equation gives us the distribution associated with the alternating harmonic series. Notice that, unlike the alternating harmonic series, it has a completely precise and unambiguous meaning as regards rearrangement of terms in the infinite sum. We claim that it makes precise the meaning of the alternating harmonic series without regards to specifying anything about term order. We consider

$$
\begin{aligned}
& \left\langle f, \varphi_{\alpha}\right\rangle=\int_{\mathrm{R}} f(x) \varphi_{\alpha}(x) d x \\
& =\int_{1}^{\infty} \sum_{n=1}^{\infty} \frac{(-1)^{n+1}}{n} \delta(x-n) e^{-\alpha x} d x \\
& =\sum_{n=1}^{\infty} \frac{(-1)^{n+1}}{n} e^{-\alpha n}
\end{aligned}
$$

By applying the monotone convergence theorem [9] to even and odd $n$ sums separately, we conclude that

$$
\lim _{\alpha \rightarrow 0}\left\langle f, \varphi_{\alpha}\right\rangle=\sum_{n=1}^{\infty} \frac{(-1)^{n+1}}{n} .
$$

If we wish, we may think of the right-hand side of eqn. (19) in a formal sense as $\langle f, 1\rangle$, and so, in a formal sense, we may view the alternating harmonic series (standard order) as $\langle f, 1\rangle$.

Using Gradsteyn and Rhysik [6] we are able to compute the absolutely convergent sum in eqn. (18). We find

$$
\begin{aligned}
\left\langle f, \varphi_{\alpha}\right\rangle & =\sum_{n=1}^{\infty} \frac{(-1)^{n+1}}{n} e^{-\alpha n} \\
& =\ln \left(1+e^{-\alpha}\right) .
\end{aligned}
$$

Taking the limit $\alpha \rightarrow 0$ of both sides of this equation and using eqn. (19), we recover the above result that the sum of the alternating harmonic series is $\ln (2)$, except, in this interpretation of it in a distributional sense there is no need for specifying the natural term order, since any rearrangement of the sum in eqn. (20) will give a result identical to that of any other arrangement.

We note that the specific form of the test function does not matter, and we have chosen to work with $\varphi_{a}$ only because of computational simplicity. We will still get eqn. (19) with any other one parameter family of functions $\varphi_{\alpha}^{\prime}$ in $S$ with the following properties:

i) $\quad \varphi_{\alpha}^{\prime}(x) \leq 1$;

ii) $\quad \varphi_{0}^{\prime}(x)=1$;

iii) $\varphi_{a}^{\prime}(x)$ monotonically decreases with increasing $\alpha$; and

iv) $\quad \varphi_{a}^{\prime}(x) \in S$.

The reason for this is due to the fact that we can always interchange the limit $\alpha \rightarrow 0$ and the summation sign in eqn. (19) for any $\varphi_{a}^{\prime}(x) \in S$ which decreases monotonically in $\alpha$ by the monotone convergence theorem. Note that for any $\varphi_{\alpha}^{\prime}$, we always have absolute convergence of $\left\langle f, \varphi^{\prime}{ }_{\alpha}\right\rangle$ and hence invariance under rearrangement of terms in the resulting infinite series.

\section{CONCLUSIONS}

According to Robert Grosso, Jr., et al. [1] in the Chemistry Department of the University of Massachusetts, in a Madelung sum each term is the sum of all electrostatic interactions among ions at a given distance from a reference ion divided by that distance, and different Madelung sums correspond to different crystal structures. The alternating harmonic series is a one dimensional Madelung sum. Because the Madelung sum naturally is conditionally convergent, it needs to be generated in a specific way in order to converge. However, with the distributional method of 
interpretation presented in this paper, the order of the terms is irrelevant. By choosing a test function, multiplying it against the distribution which represents the conditionally convergent series, summing the terms, and then letting $\alpha \rightarrow 0$, an unambiguous meaning has been assigned to conditionally convergent series. This allows them to handled easily in the physical situations where they appear. As the above example shows, this method not only serves as an aid in a computational approach to calculating infinite series, it also serves to make precise otherwise confusing notions about infinite series which are embodied in the result of Riemann.

\section{REFERENCES}

1. Robert P. Grosso, Jr., Justin T. Fermann, and William T. Vining, "An InDepth Look at the Madelung Constant for Cubic Crystal Sums," Journal of Chemical Education 78 (2001), pp. 1198-1202.
2. Walter Rudin, Principles of Mathematical Analysis, International Series in Pure and Applied Mathematics, $3^{\text {rd }}$ Edn. (McGraw-Hill, Inc., New York, 1976).

3. George Arfken and Hans J. Weber, Mathematical Methods for Physicists, $6^{\text {th }}$ Edn. (Elsevier Science \& Technology Books, Utrech, the Netherlands, 2005).

4. en.wikipedia.org/wiki/harmonic_series $\%$ 28mathematics $\% 29$

5. Larry Riddle, "Rearranging the Alternating Harmonic Series" Web publication at: ecademy.agnesscott.edu/ Iriddle/series/rear.htm.

6. I.C. Gradshteyn, I.M. Rhyshik. Tables of Integrals, Sums, Series and Products (Academic Press, New York, 2000).

7. en.wikipedia.org/wiki/EulerpMascheroni constant

8. Robert S. Strichartz, A Guide to Distribution Theory and Fourier Transforms (Academic Press, New York, 2006).

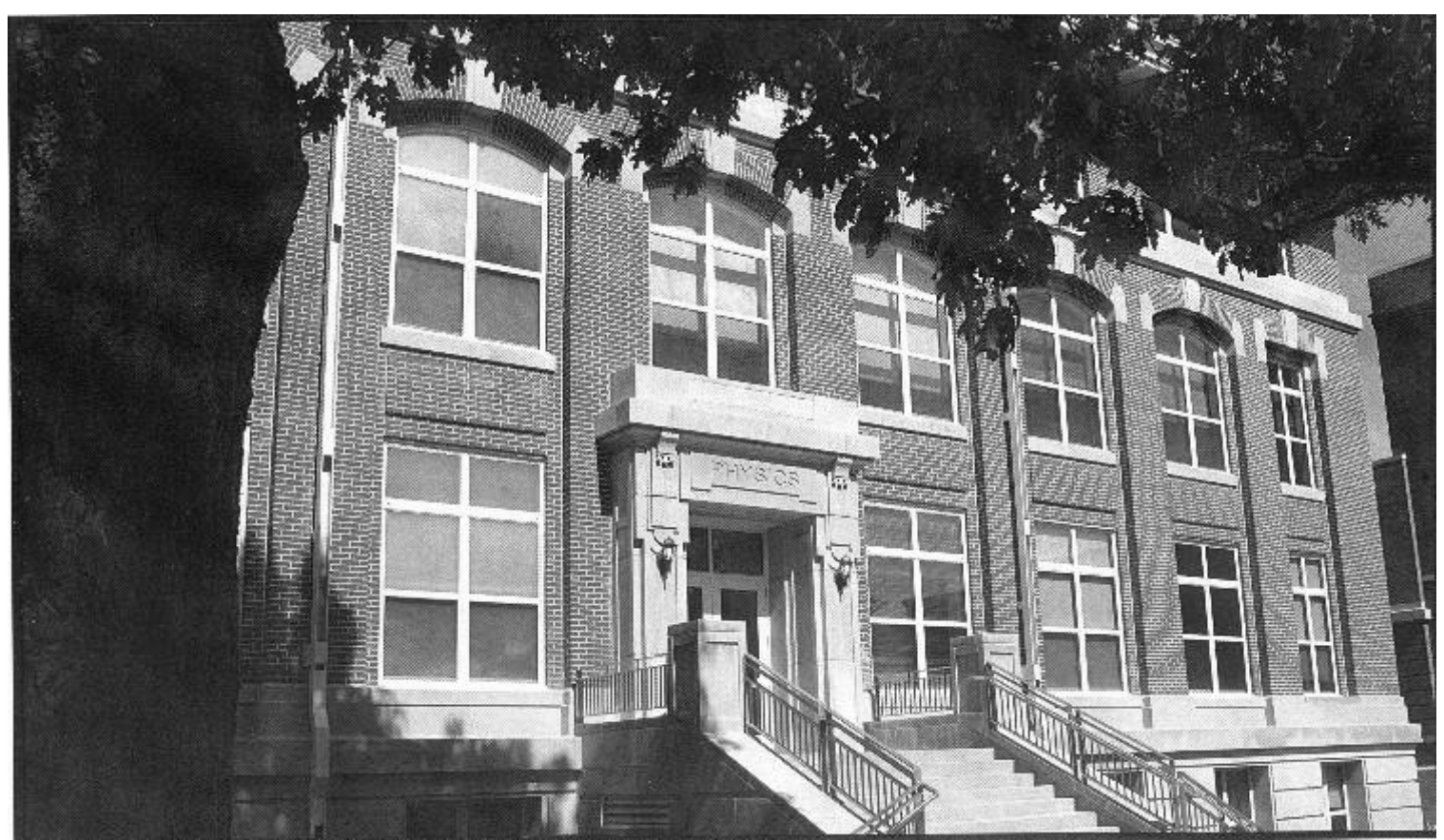

Begeman Hall $\downarrow$ Department of Physics at the University of Northern lowa • Cedar Falls, lowa 50614-0150 USA • www.uni.edu/physics

A national leader in physics education at both the undergraduate and master's levels. 


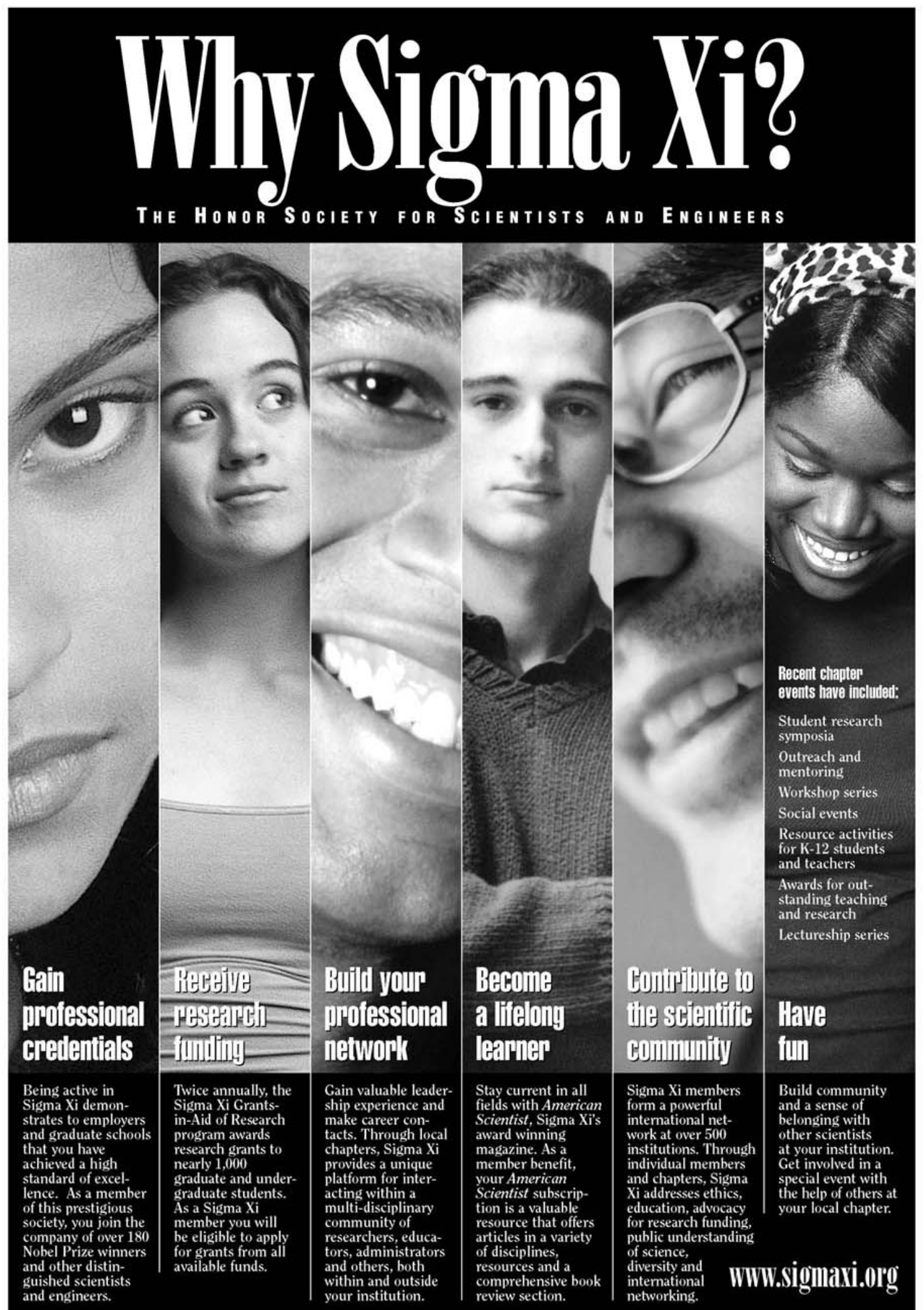

Sigma Xi, The Scientific Research Society • 98 Alexanter Drive • P.O. Box 13975 • Research Triangle Park, NC 27708 • 818-549-4691 • 800-243-6534 